\title{
The Concept of Fifth Generation (5G) Mobile Technology
}

\author{
Khalid A. Fakeeh, PhD \\ King Abdullaziz University \\ Jeddah, Saudi Arabia
}

\begin{abstract}
In the present day 3rd Generation (3G) mobile systems are in progress for endowing Internet Protocol (IP) service for instantaneous/synchronized and non-instantaneous services. In contrast, loads of wireless technologies have demonstrated and turned out to be significant and out of them one which is extremely vital is 802.11 "Wireless Local Area Networks" (WLAN) and 802.16 "Wireless Metropolitan Area Networks" (WMAN), with the possible addition of ad-hoc Wireless Personal Area Network (WPAN) and wireless networks for digital television and radio transmission. The communication capacities with community on the shift have astonishingly progressed. With each momentary generational rise in the employment of mobile communications systems, the integrated elucidation of communication with mobility has reidentified and transfigured the way of the communication of people by guarantee connectivity in progress ubiquitously. In a comparatively squat extent of time, wireless entrée technologies have progressed and got to its fourth generation (4G) with side by side delve into preparing itself for the exploitation of the novel or innovative fifth generation (5G) technology. This research article outlines the concept of 5th Generation Wireless technology.
\end{abstract}

\section{Keywords}

Wireless Communication, 4G, 5G

\section{INTRODUCTION}

In the last few years wireless and mobile networks have turned out to be remarkable development. At the moment a lot of mobile phones have also a WLAN adapter. One may possibly believe that in near future countless mobile phones will have WiMAX adapter too installed in it, in addition their Bluetooth, 3G, 2G, WLAN etc. adapters.

$5 \mathrm{G}$ technology will possibly revolutionize the approach the majority lofty bandwidth customer's right to use their mobile phones. With a device having VOIP enabled service and the service $5 \mathrm{G}$, individuals will experience and enjoy an intensity of call volume and data communication which they have by no means practiced before. This technology is putting forward its services in Documentation, Product Engineering, sustaining electronic transactions (e-transactions, e-Payments) and so on. Since the client turn out to be more and more conscious in regards of mobile phone technology, they will definitely come across for an upright package collectively, comprising all the sophisticated facets a cellular phone be capable of or possess. For this reason the exploration for newfangled technology is for all time the core aim of the top cell phone colossal to out innovate their opponents. Lately apple has trembled all around the electronic globe by initiating its innovative handset/phone so called the I-phone. Characteristics that are in receipt of implanted in such a minute piece of electronics are enormous. The $5 \mathrm{~g}$ devise/design has a user-centric mobile based environment/setting with numerous mobile and wireless technologies. As far as the $4 \mathrm{G}$ technology is concern so its main concern is towards faultless Bluetooth and incorporation of cellular/mobile networks e.g. WLAN, GSM and 3G. In case of $4 \mathrm{G}$ multimode client terminals are must, however diverse safety methods and unusual Quality-of-Service (QoS) hold up in dissimilar wireless technologies hang about a defy. On the other hand, AAA incorporation amid unlike wireless networks such as WLAN and PLMN is carrying out its practice even in the present day. In [6] the projected Open Wireless Architecture (OWA) is besieged to endow with unbolt baseband processing components with unwrap interface strictures or factors to hold up dissimilar on hand with addition of prospect wireless communication standards/archetypes.

\section{4G OVERVIEW}

In this section we will out the characteristics of $4 \mathrm{G}$ technology to give an idea, that why it is still so vibrant to going on with.

\subsection{Favorable Performance and Capabilities}

Engineering professional articulate that customer's of $3 \mathrm{G}$ will not be able to take full fledge benefits of rich multimedia content of it. As far as $4 \mathrm{G}$ is concern so it will trait exceptionally lofty quality of video as good as to HD Videos. Average speed of wireless downloads having speed reaches to $100 \mathrm{Mbps}$, which is 50 times of $3 \mathrm{G}$, and are probable with that of $4 \mathrm{G}$.

\subsection{Trouble-free Roaming and Straightforward Interoperability}

Interoperability across various networks in $3 \mathrm{G}$ technology is difficulty just because of it manifold standards. In contrast $4 \mathrm{G}$ endow with an international standard that is capable of endowing an international mobility. An assortment of diverse wireless access networks on average diverge in provisions of data rate, coverage, loss rate and coverage. For that reason, they are sensibly intended to hold up a special set of explicit devices and services. $4 \mathrm{G}$ will take in different sorts of terminals, which may possibly have to afford widespread services autonomously of their qualifications. This perception is known as service personalization.

\subsection{Effusive Coupled Services}

The customer of $4 \mathrm{G}$ can access any sort of application from unusual platforms e.g. Laptops, Cell Phones or anything else, for the reason that $4 \mathrm{G}$ is able to endow a sharp connectivity and is competent enough to hold up video streaming, VOIP, email, web browsing, e-commerce and so on. Which is so called a freedom of choice for users.

\subsection{Squat Outlay}

$4 \mathrm{G}$ is in a position to prove that it's extremely cheaper than the conventional $3 \mathrm{G}$ systems, in view of the fact that they be able to be built atop on hand networks and is not able to entail 
operators, carriers to utterly retool and acquire expensive additional spectrum. Furthermore an assortment of additional outlay professional, $4 \mathrm{G}$ is spectrally well-organized, so carriers are in position to achieve alot with fewer.

\subsection{User-Friendly Interface}

The devices of $4 \mathrm{G}$ technology are projected to be extremely visual and spontaneous as compared to the present day menu and text based systems. They are sufficient able to act together with the setting in its region and proceed consequently.

\subsection{Improved GPS (Global Positioning System) Services}

Furthermore $4 \mathrm{G}$ has an enhanced GPS system to locate individuals and Location and may possibly be able to permit public be practically nearby in an assortment of places.

\subsection{Expandable/Scalability}

Scalability is one of the most exigent facets of the cellular networks. It passes on to aptitude to grip constantly growing number of customers and services. In view of the fact that all IP center layer of $4 \mathrm{G}$ is effortlessly scalable/expandable and it is preferably well-matched to convene this defy.

\subsection{Disaster-Management Applications}

Natural catastrophe is capable of distressing the whole communications structural design is in dismay. Re-establish communications speedily is indispensable. By means of wideband wireless mobile communications Internet and video services, may possibly be launched in hours rather than of days or even weeks obligatory for re-establishment of wired transmissions.

\section{MIGRATION DEFIES:}

\subsection{Multimode user terminals:}

In the company of $4 \mathrm{G}$ technology there will be a call for planning a solitary user/customer terminal that will be able to maneuver in unusual wireless networks and prevail over the design/devise predicaments e.g. device size constraints, outlays and power utilization. This dilemma can be work out by utilizing software radio practice.

\subsection{Selection of Wireless System}

By far and large each and every wireless system has its own inimitable distinctiveness and responsibility. The propagation or flare-up of such technologies set hurdles in selecting or choosing the most appropriate technology for a meticulous service at a picky time and position. Selecting a system according to the user Quality-of-Service requirements and inhand resources can sort out this problem.

\subsection{Safety Measures \& Protection}

Diverse variety and an assortment of wireless networks make matters worse regarding the security concern. Self-motivated adaptive, re-configurable, and inconsequential defense methods/practices ought to be fashioned.

\subsection{Structural Design and Quality of Service Hold up}

Incorporating the offered non-IP systems/designs and IPbased systems and endowing Quality of Service (QoS) pledge for end-to-end services that engross unusual systems is also an immense defy.

\subsection{Billing Information Concerns}

It is almost or extremely wearisome to pull together, handle and lay up the user's accounts information from manifold service suppliers.

\subsection{Application Level Molests}

$4 \mathrm{G}$ mobile wireless devices is well recognized for software applications which will endow with a ground-breaking facet to the customer other than will bring in up-to-the-minute holes, directing towards more molests/assails at the application stage.

\subsection{Spoofing and Overcrowding Dilemma}

The term spoofing passes on to counterfeit GPS signals being transmitted, in regards to that the GPS receiver assumes that the receiving signals approaches from a satellite and determines the erroneous coordinates. Scandalous can bring into play such methods to obstruct with law exertion. The other term overcrowding comes about when a source sending out signals at the identical frequency put out of place a concern GPS signal.

\subsection{Call for an Enhanced Data Encryption Techniques}

If a GPS receiver wants or need to be in touch with the central transmitter/source, in that case the communication link flanked by these two components is not inflexible to shatter and there is a call for utilizing encrypted data.

\section{5G CONCEPTS}

Terminals of $5 \mathrm{G}$ will be comprising software defined radios (SDR) and modulation methods or designs with addition of new-fangled error-control methods that is available to be downloaded from the Internet quite easily. The growth is seen in the direction of the user terminals with respect to $5 \mathrm{G}$ mobile networks. The terminals are competent enough to afford access to diverse wireless technologies concurrently and the terminal ought to be competent enough to mingle unusual flows from dissimilar technologies. The upright tenders ought to be circumvented, for the reason that they are not realistic in the case of presence of various technologies, operators and service suppliers. Each network will be accountable for managing user-mobility, at the same time as the terminal will construct the ultimate preference surrounded by unusual wireless/mobile access network suppliers for an agreed service. And such concern selection will be based on unbolt sharp middleware in the mobile phone. Subsequently all OSI Model layers in the $5 \mathrm{G}$ mobile terminal will be discussing.

\subsection{Physical \& MAC Layers}

These are the top layers of OSI model and they characterize the wireless technology. 5G mobile networks are expected to be based on Open Wireless Architecture for these two layers[6].

\subsection{Network Layer}

This layer often refers to an IP (Internet Protocol) layer, for the reason that there is no rivalry in the present day on this point. The IPv4 is all-inclusive broaden with having numerous defies like restricted address space and has no authentic likelihood for Quality-of-Service hold up per stream. These concerns are worked out in subsequent IPv6, but in business with notably superior packet header. Followed by the problem of mobility, we have Mobile IP standard/archetype on one 
side with addition of numerous micro-mobility elucidations. Mobile IP will be utilized by all mobile networks in 5G, and every mobile terminal will be an FA referred as a Foreign Agent, holding up the "Care of Address" (CoA) mapping flanked by its predetermined IPv6 address and "Care of Address" for the contemporary wireless network. On the other hand, a mobile is able to be affixed to quite a few wireless or mobile networks concurrently. In such a scenario, it will uphold unusual IP addresses for all of the radio interfaces, whereas each of these IP addresses will be CoA address for the FA positioned in the mobile Phone. The 5G phone manufactures will put into action the unchanging IPv6 in the mobile phone. Implicit multi-wireless network setting shall be kept up by the $5 \mathrm{G}$ mobile phone. In view of this fact there ought to be severance of network layer into two sub-layers in $5 \mathrm{G}$ mobiles. This is just because of the preliminary devise of the Internet, where majority all of the routing IP based and which ought to be diverse in each IP network all-inclusive. The middleware between the two sub-layers shall uphold address translation from Upper network address (IPv6) to dissimilar Lower network IP addresses (IPv4 or IPv6), and vice versa.

\subsection{Open Transport Protocol (OTA) Layer}

Wireless and mobile networks can be differentiated from the wired network on the basis of transport layer. In the entire TCP versions the postulation is that mislaid sectors are just because of network obstruction, whereas in wireless networks sufferers may possibly come about just because of privileged bit error ratio in the radio interface. For that reason, TCP amendments and alteration are projected for the mobile and wireless networks, which retransmit the vanished or smashed TCP segments over the wireless link merely. On behalf of 5G mobile terminals will be fitting to encompass transport layer that is probable or likely to be downloaded and settled up. Such mobiles shall have the likelihood to download e.g., RTP, TCP or new-fangled transport protocol version which is besieged to an explicit wireless technology initiated at the base stations referred here as an Open Transport Protocol OTP.

\subsection{Application Layer}

On the subject of the applications, the decisive appeal from the $5 \mathrm{G}$ mobile terminal is to endow with a smart Quality-ofService administration above assortment of networks. In the present day, the customers in the mobile phones manually opt for the wireless interface for meticulous Internet service devoid of having the likelihood to utilization Quality-ofService history to decide on the unsurpassed wireless connection for an agreed service. The $5 \mathrm{G}$ phone out to be in a position to endow with prospect for service eminence taxing/testing and storage of extent information in databases in the mobile terminal. The Quality-of-Service constraints e.g. impediment, jitter, fatalities, dependability, bandwidth will be accumulated in a $5 \mathrm{G}$ mobile phone database with a purpose ought to be utilized by intellectual algorithms successively as system processes in the mobile terminal, shall afford the unsurpassed wireless connection leading mandatory Qualityof-Service and individual outlay constrictions. In the company of 4G, an assortment of innovative services and sculpts will be on hand. Which ought to be additional scrutinized for their interface with the devise of $4 \mathrm{G}$ systems. The course of IPv4 address overtiredness is likely to be in its ultimate phases by the time that $4 \mathrm{G}$ is set up. For that reason, IPv6 hold up for $4 \mathrm{G}$ is indispensable in order to prop up an outsized quantity of wireless- supported devices. IPv6 gets rid of the call for NAT
"Network Address Translation" by mounting the number of IP addresses. By means of the accessible address space and number of addressing bits in IPv6, numerous ground-breaking coding techniques can be fashioned for $4 \mathrm{G}$ applications and devices that could lend a hand in the exploitation of $4 \mathrm{G}$ services and network. The $4 \mathrm{G}$ guarantees to accomplish the aspiration of PCC "personal computing and communication", hallucination that affordably endows towering data rates all over the place over a wireless network [3]. In the prospect regards to wireless networks there have got to be a squat intricacy of realization and a well-organized conciliation amid the wireless infrastructure/structural design and end users. For superior data rates and lofty speed entrée to the mobile wireless users, Internet is the striving strength. This will be the impetus for the entire IP based center network progression.

\section{THE STRUCTURAL DESIGN OF 5G}

$5 \mathrm{G}$ is in progress to have room for the Quality-of-Service and rate necessities lay down by approaching applications similar to Multimedia Messaging Service (MMS), wireless broadband access, video chat, HDTV content, mobile TV, Digital Video Broadcasting (DVB), negligible services approximating voice and data, and further services that make use of bandwidth. The classification of $5 \mathrm{G}$ is to make available satisfactory Radio Frequency coverage, additional bits $/ \mathrm{Hz}$ and to be linked all wireless diverse networks to endow with faultless, reliable telecom practice to customer.

\subsection{The Evolved Packet Core (EPC)}

It is flat IP network theory based and an IP-based core/central network characterized by 3GPP a Telecom standard for utilization by LTE (Long Term Evolution) and further entrée technologies. EPC objective is to afford cut down entire IP core network structural design to competently bestow way in to an assortment of services. It comprises in essence of an access agnostic gateways for routing of user datagram and Mobility Management Entity (MME). It will be a absolutely new-fangled architecture/structural design for wireless operators/suppliers to imitates the IP world of data Communication rather than the voice- centric world of wireless.

\subsection{FLAT IP STRUCTURAL DESIGN}

Mobile networks have been planned till this position for circuit, switched, voice. Wireless networks were intended in a hierarchal manner to amassed, substantiate, handle and express calls. A BSC combines calls from manifold base stations, allots radio channels, facilitates handoffs flanked by base stations and outdo on calls to more centralized mobile switching centre. After packet data networks came into view, they were superimposed on the offered voice-centric structural design, utilizing the BSC for the identical mobility administration purpose and toting up the GGSN and a PDSN in the case of CDMA and SGSN in the case of GSM/UMTS to course and administer data sessions, plus to hook up to the Internet or suitable IP network. There is a rising factor in data traffic. This voice centric structural design has turned out to be weighty and severe to deal with too countless network entity/units. It eradicates that voice-centric hierarchy from the network. As a substitute of superimposing a packet data core on the voice network, break up and greatly cut down data structural design can be put into operation that get rid of the manifold rudiments from the network succession. BSC roles are alienated among media gateway router and Base station. Base station will commune unswervingly by means of 3GDT 
(3G direct tunnel) by way of media gateway over WAN. A number of the roles of BSC/RNC will be griped by base stations. This practice has undoubtedly noticeable compensations. By dipping the network hops, data pass through quicker amid end points, significantly tumbling the network latency to lend a hand to hold up real-time applications. The emergence of the IP architectures/structural design came into existence with WiMAX, and prospect LTE (Long Term Evolution) networks will be flat by characterization.

\section{SUMMARY}

The $5 \mathrm{G}$ mobile phone is intended as an unbolt podium on unusual layers, starting from physical layer up to the application. The future perception acclimatizes Open Wireless Architecture projected for $4 \mathrm{G}$ mobile terminals, and affords additional alterations from network up to the concern application layer. The two sub-layers of network are intending to endow with all-IP connectivity in surroundings with profusion of wireless/mobile technologies and network service suppliers. Open Transport Layer is projected with endeavor to tolerate practice of wireless explicit realizations of transport protocols. In conclusion, there is an opportunity for assortment of unusual wireless technologies upon diverse Quality-of-Service constrictions for dissimilar services and for such reason, 5G mobile terminals ought to keep up database to maintain statistical information concerning the services and the existing wireless technologies. At present, the current work is on the components/units that shall endow with the unsurpassed Quality-of-Service and lowly outlay for a specified $5 \mathrm{G}$ service by means of one or more wireless technology concurrently.

\section{REFERENCES}

[1] "T.Janevski", "A System for PLMN- WLAN Internetworking", Journal of Communications and Networks (JCN), pp.192-206, Vol 7, No. 2, June 2005.

[2] "J.McNair", "Vertical Handoffs in Fourth-Generation Multinetwork Environments", IEEE Wireless Communications, June2004.

[3] "T.Janevski", "Traffic Analysis and Design of Wireless IP Networks", Artech House Inc., Boston, USA, 400 p.,
May 2003.

[4] "Kai Hau Yeung, Suk Yu Hui,", "Challenges in the Migration to 4G Mobile Systems", IEEE Communications Magazine, December 2003.

[5] "O. Queseth, R, F. Gessler, A. Bria, J.Wu, J.Zendler", "4-th Generation Wireless Infrastructures: Scenarios and Research Challenges", IEEE Personal Communications, Vol. 8, No. 6, December 2001.

[6] "W.W. Lu", "An Open Baseband Processing Architecture for Future Mobile Terminals Design", IEEE Wireless Communications, April 2008.

[7] "J.Govil", "5G : Functionalities development and an Analysis of Mobile Wireless Grid", First International Conference on Emerging Trends in Engineering and Technology.

[8] "Frank H.P ,Simone Frattasi,", Aalborg University; Marcos D. Katz, Samsung Electronics, "Defining 4G Technology from the User's Perspective." IEEE Network January/ February 2006.

[9] "K.R.Santhi", "Migration to 4 G: Mobile IP based Solutions" Proceedings of the Advanced International Conference on Telecommunications and International Conference on Internet and Web Applications and Services (AICT/ICIW 2006).

[10] "R.Berezdivin, R Topp, Raytheon", "Next- Generation Wireless Communications Concepts and Technologies" 0163- 6804/02/2002 IEEE, IEEE Communications Magazine, March 2002.

[11] "E.Aravantinos M. Hosein “., Wesley J. Howe School Of Technology Management, Stevens Institute Of Technology, Hoboken, NJ 07030 "Potential Scenarios And Drivers Of The 4g Evolution".

[12] "H-Hwa Chen, R Fantacci", University Of Firenze, "The OCC- CDMA/Os For 5G Wireless", IEEE Vehicular Technology Magazine ,September 2006. 